\title{
Renal Transplantation and Aortic Disease: Operative Management
}

\author{
Gabriele Piffaretti, Matteo Tozzi, Marco Franchin, \\ and Patrizio Castelli
}

\subsection{Introduction}

In the last decade, we recognized three main facts that dramatically changed the perspective on kidney transplant. Firstly, progress in surgical technique and the development of new immunosuppressant protocols have lengthened graft survival; secondly, population affected by end-stage kidney disease (ESKD) has become even older; and finally, renal transplant has increased the longevity of graft recipients. As a result, kidney transplant recipients (KTRs) are more and more exposed to chronic degenerative pathologies, such as atherosclerosis. Additionally, it is necessary to emphasize the relationship existing between ESKD, long-term hemodialysis, and accelerated atherosclerosis [1]. Subsequently, an increase in the prevalence of aortic atherosclerotic disease, either occlusive or dilatative, in KTR must be expected.

It is remarkable how Brekke et al. in 1993 reported a prevalence of both aneurysmal and occlusive diseases in only $1.86 \%$ of patients waiting for kidney transplant. Accordingly, in a literature review published in 1994, Panneton et al. described the concomitance of aortic aneurysm and kidney transplant as a very rare event and reported only 51 cases of aortic recon-

G. Piffaretti · M. Tozzi · M. Franchin $(\bowtie) \cdot$ P. Castelli Vascular Surgery, Department of Medicine and Surgery, Circolo University Teaching Hospital, University of Insubria School of Medicine, Varese, Italy struction performed in organ recipients in the previous 30 years [2]. Nowadays, according to the Michigan Transplant Centre Registry, prevalence of aortic aneurysm between KTRs has increased to $4 \%$ [3].

In a review by Droupy et al. on 1110 kidney transplants, a severe occlusive disease affecting iliac arteries was documented in $9.6 \%$ of KTR. Among them $3.4 \%$ required endarterectomy at the time of the transplant. In analyzing follow-up data, they concluded that overall graft survival was significantly lower if compared to patients without iliac atheroma [4-6].

It means that in the future, a new challenge for vascular and transplant surgeons will be represented by the aortoiliac reconstruction in KTR.

The management of many problems associated to aortic surgery needs to be solved in prospective, preoperative evaluation and screening, risk stratification and surgical planning, graft protection from ischemia and reperfusion injury during aortic surgery, and follow-up.

\subsection{Consideration on Open Surgery in Kidney Transplant Recipients and Graft Protection Techniques}

Sehti et al. in 1976 were the first to report a case of thoracic aorta repair in a KTR for a ruptured type B dissecting aneurysm [6]. Soon after, Shons 
et al. and Sterioff et al. reported two cases of abdominal aortic aneurysm repair in KTR [7, 8]. Unfortunately, the three patients died after intervention, and the first to report a successful abdominal aortic aneurysm repair with a femorofemoral partial extracorporeal circulation was Campbell in 1981 [9].

Open surgery for the management of aortic aneurysms in KTR presents various problems. The first and most relevant is that renal graft derives blood supply from the iliac artery below the aneurysm. Consequently, aortic repair can be responsible for transient or permanent ischemic injuries and even graft loss. Second, the previous kidney transplant can be associated with perivascular fibrosis and consequently difficulty in dissecting vessels and higher risk of graft parenchyma and ureter damage.

Graft protection technique continues to be the main and open question in literature. The options commonly adopted by surgeons for graft protection are:

- No protection

- Topical cooling with sterile ice

- In situ graft cold perfusion (static or dynamic perfusion technique)

- Ex situ graft cold storage (static storage or dynamic perfusion technique)

- Distal aortic perfusion with a shunt or bypass

- General hypothermia

The technical choice should be influenced by many factors including:

- Graft fitness

- Complexity of aortic repair

- Estimated duration of kidney ischemia

- Confidence with a specific preservation technique

Adjunctive renal protection measures must be taken into account in case of difficult surgical anatomies including arterial graft anastomosis on large iliac aneurysms and impaired graft function (creatinine serial level $>250 \mu \mathrm{mol} / \mathrm{L}$ ).

Unfortunately, literature mainly reports case reports or small series concerning personal author experience. The analysis of these results does not demonstrate the superiority of a particular technique. A "clamp and go" technique without graft protection and short cross-clamp time can be considered a safe alternative in an experienced hand. Otherwise, the protective technique can prolong operative time and can be prone to graft damage, thrombosis, or sometimes ischemic injury if not done correctly.

\subsubsection{None Graft Protection}

During the 1970s, when this surgery was first developed, it was postulated that aortic clamping would be responsible for total graft warm ischemia [7, 8]. Otherwise, with experiences conducted between 1973 and 2005, Lacombe demonstrated that during the aortic clamp, the graft continued to be perfused by the retrograde flow from the lumbar, inferior mesenteric, and both iliac arteries. Lacombe proved that during aortic clamp the back pressure through the collateral pathway is always $>35 \mathrm{mmHg}$ [10], while Morris et al. showed experimentally that the minimum pressure needed for kidney viability is $25 \mathrm{mmHg}$ [11]. Accordingly, total graft ischemia would occur only during renal artery reattachment when necessary. However, it has been demonstrated that the kidney can tolerate periods of total artery occlusion of up to $50 \mathrm{~min}$, more than the time required for anastomosis [12]. Lacombe reported a successful experience conducted on 18 KTRs without the aid of vascular shunts. When the surgeon does not adopt a preservation method, Lacombe suggested to apply small but substantial modifications to the standard surgical technique. Particularly, he recommended to make a suprarenal transverse section of the aorta between two clamps followed by an end-to-end anastomosis of the prosthesis to the aorta. The second step is represented by the revascularization of the kidney by the prosthetic graft anastomosis on the iliac axis proximal to the transplant artery or by the renal artery reattachment on the prosthetic graft. The third step is the management of the aneurysmal sac and the opposite limb revascularization [10]. 


\subsubsection{In Situ Cold Perfusion and Ex Situ Preservation}

Many authors suggested that in situ static cold perfusion with Ringer's lactate $\left(4^{\circ} \mathrm{C}\right)$ through the common iliac artery combined with topical cooling may be the faster and easiest alternative for graft preservation [13]. More recently, Tshomba et al. showed better results in terms of perioperative renal function after aortic clamp with Custodiol over Ringer's lactate [14]. Alternatively, our experience suggests that ex situ static cold storage with a standard preservation solution (Celsior or University of Wisconsin solution) and the subsequent re-transplantation of the organ on the prosthetic graft represent a safe, effective, and viable alternative. The main advantages offered by this technique are comfort and the possibility of extending the surgical time without substantial risk of compromising residual renal function. Possible complication is represented by the risk of renal artery dissection during cannulation and perfusion, especially in case of marginal graft. Additionally, we must remember possible complications related to cold injury and reperfusion (see Chap. XXX).

\subsubsection{Distal Aortic Perfusion with Shunt or Bypass}

A wide range of techniques have been described in literature. Mostly, we can group them between axillo-femoral and aortofemoral shunts.

Shons et al. first reported the use of axillofemoral permanent bypass with an $8 \mathrm{~mm}$ woven Dacron graft. The aorta was ligated distally to the renal artery and common iliac artery and proximally to the origin of hypogastric. Graft anastomoses were performed on the external iliac vessels [7]. Temporary axillo-femoral shunts were described by Gibbons et al. and Roach et al. with satisfactory results in five patients. They admitted an increase in operating time, but deterioration in renal function was not seen in any case $[15,16]$.

More frequently, temporary aortofemoral bypass with Gott shunt has been described [17,
19]. Other authors have adopted polyvinyl tubes as the shunt with optimal perfusion pressure (only $15 \mathrm{mmHg}$ less than systemic pressure) maintained for long cross-clamp times [8, 18]. Finally, successful results have been obtained with modified techniques, adopting an inline shunt instead of separate aortic cannulation [19].

\subsection{Endovascular Aortic Reconstruction}

A safe and effective alternative to open surgery is represented by endovascular reconstruction for both aneurysm and aortic occlusive diseases. This surgery was proposed for the first time in 2000 by Sawhney et al. in the treatment of abdominal aortic aneurysms with aorto-uni-iliac right graft and femoro-femoral crossover bypass to perfuse a left-sided renal transplant and homolateral lower limb [20]. In 2001, Forbes described the first treatment of a large aneurysm with an endovascular approach bifurcated graft [21]. Endovascular aortic repair (EVAR) demonstrated two main potential advantages if compared to open conventional surgery. Firstly, aorta and iliac cross clamping is not required, minimizing ischemic risk. Additionally, in cases of abdominal aortic aneurysms, it is not necessary to take into account the limitations associated with proximal neck morphology and length (pararenal and juxta-renal aneurysms) because of the possibility of proximal extension [22]. Actually, tube graft use is limited to those cases that are characterized by severe, not crossable, atherosclerosis of the common iliac artery. Bifurcated devices are largely used. The main body must be introduced through the contralateral iliac artery, while the lower profile limb is delivered from the donor iliac artery. It is not actually clear if the newer lower profile prosthesis has better results in terms of complications over conventional grafts. In fact, potential risks associated to endovascular procedures may be renal graft or vessel iatrogenic lesions. In a recent review of 17,213 cases of EVAR for abdominal aneurysm from the Vascular Quality Initiative dataset, Bostock et al. did not find any difference 
in technical outcomes between KTR and conventional patients (KTRs were $0.2 \%$ of all patients). Endoleak occurred in $20 \%$ of KTR $(p=0.46)$, while no graft migration and infection were seen [23]. According to everyday experience, limitations to endovascular aortic procedures remain femoro-iliac atheromasia and obstinate stenosis that can obstacle the sheath progress. Additionally, large delivery systems can produce vascular lesions, plaque dissections, or renal graft artery damage. For that reason, the manipulation of catheters and wires should be kept at a minimum. Large-bore delivery systems and sheaths must be placed downstream of the orifice of renal graft artery, and they should be removed as soon as possible to avoid unintentional artery occlusion or damage. Surprisingly, Bostock et al. reported a very low incidence of arterial injuries requiring open repair with no statistical difference between the two cohorts $(p=0.62)$. In every case, surgical correction was not related to kidney dysfunction. Finally, in their large review, they did not describe any event of kidney graft damage or renal artery lesions.

Another possible complication related to endovascular aorta reconstruction in KTRs is contrast-induced nephropathy. According to literature, transplanted kidneys and autogenous kidneys are prone to contrast-induced nephropathy in the same way [24, 25]. Once again, Bostock et al. seem to contradict what was previously reported in literature. In fact, they showed that renal dysfunction defined as acute kidney injury (elevation of serum creatinine $0.5 \mathrm{mg} / \mathrm{dL}$ ) or necessity of postoperative hemodialysis was three times more frequent in KTRs over conventional patients $(p=0.02)$. They saw a significant prevalence of renal dysfunction in patients with lower glomerular filtration rate before EVAR [27]. Patient hydration, diuresis stimulation, and antioxidant agents as $\mathrm{N}$-acetylcysteine have been largely recommended [25-27]. Additionally, it is suggested to reduce the contrast dose to a minimum. Comprehensive procedure planning and correct $\mathrm{C}$-arm angulation help to minimize the contrast amount [27]. Other advanced imaging modalities, such as image fusion technology, have been described as alternatives to standard fluoroscopy [28, 29]. Bostock et al. did not find any difference in terms of absolute iodinate contrast dose in KTRs that developed postoperative nephropathy and not. However, KTR with renal dysfunction received a significantly higher dose of contrast in relation to the preoperative glomerular filtration ratio (iodine/GFR ratio 0.78 vs $0.39, p=0.02$ ) [22]. This remark should be taken into account when considering aortic repair in KTRs.

\subsection{Final Remarks: Aortoiliac Reconstruction Prior, Concomitant, or After Transplant}

Galazka et al. described an experience of over 1553 patients where $201 \quad(13 \%)$ required intervention for aortoiliac atherosclerosis. Surgery consisted in iliac endarterectomy and anastomosis of the renal graft to the hypogastric artery when patent. They also described the use of Y-shaped ilio-iliac vascular graft for iliac revascularization and kidney anastomosis [4]. Tsivian et al. reported that in their series of 1554 transplants, aortoiliac surgery was necessary in $2 \%$ of cases, and among them $80 \%$ of lesions were discovered intraoperatively [30]. Many other papers have been published on the same topics, and we can argue that intervention schedule is not usually the result of careful planning but rather the consequence of casual intraoperative findings. Piquet and Gouny investigated the correct timing for aortoiliac reconstruction in KTRs. They concluded that concomitant procedure, sometimes involving prosthetic grafts, had higher risks of infections. Consequently, they suggested delaying renal transplantation to at least 6-12 weeks after vascular reconstruction [31-32]. On the contrary, Coosemans et al. in a prospective trial compared different interventional timing, concluding that transplant concomitant to reconstruction is safe and effective, is cheaper, and has fewer difficulties and risks of graft injuries [33]. Tsivian et al. confirmed that concomitant reconstruction and transplant is safe, even if it would be preferable to 


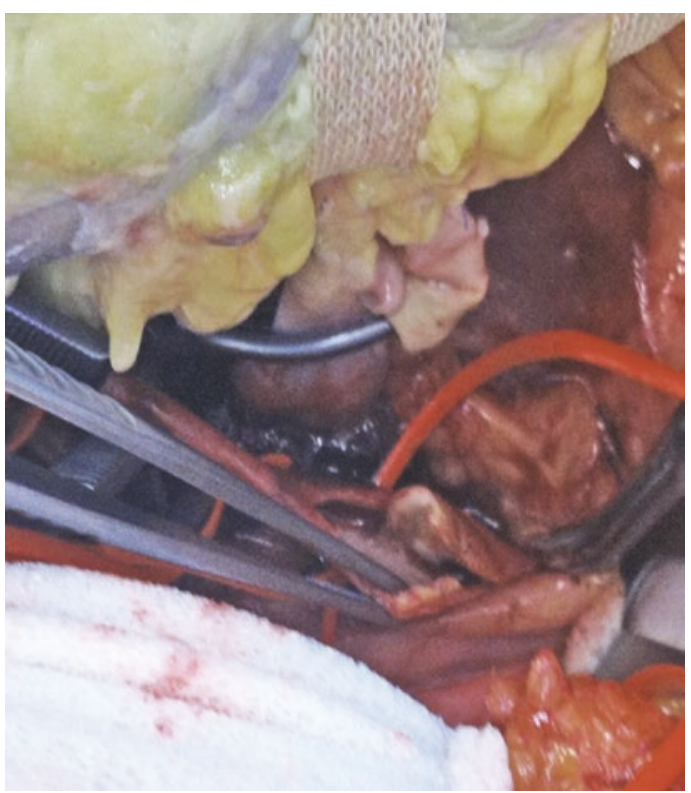

Fig. 11.1 Aortic and common iliac artery endarterectomy before kidney transplant

anticipate the aortoiliac surgery [30] (Fig. 11.1). Possible benefit could be obtained using autologous grafts instead of synthetic prosthesis, and consequently, the single-stage surgery is preferable (Figs. 11.2 and 11.3). Additionally, endovascular treatment had better results in terms of infection in comparison to prosthetic grafts.

Gill et al. suggested that proper planning cannot be separated from a correct diagnostic study. They recommended Doppler ultrasound screening in patients at high risk for atherosclerosis disease. When physical examination or Doppler is suggestive of a disease, completion angiography should be performed [34]. Tozzi et al. recommended performing CT angiography scan in all patients on the waiting list for kidney transplant with risk factors for atherosclerosis. They suggested aortoiliac stenosis can be treated simultaneously with kidney transplant. It is important to be mindful of the risk that atherosclerosis lesions could increase, therefore worsening both graft and limb vascularizations (Fig. 11.4). On the contrary, both symptomatic stenosis and aortic aneurysm (especially $>5 \mathrm{~cm}$ in diameter) should be treated before transplant, in order to reduce complications while on the waiting list. In par-

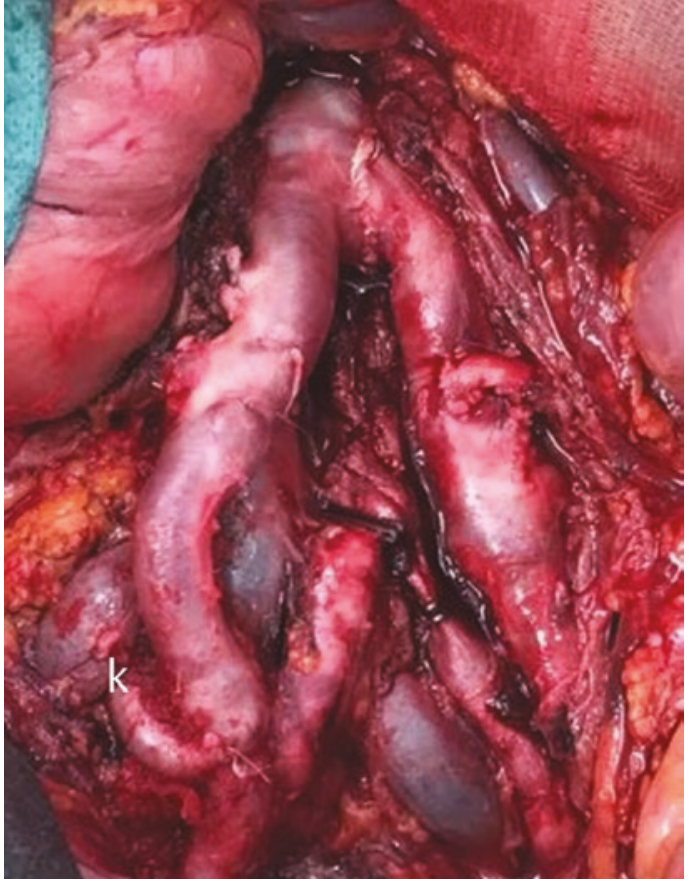

Fig. 11.2 Aorto-bisiliac composite cadaveric homograft bypass and concomitant kidney graft $(\mathrm{k})$ transplant

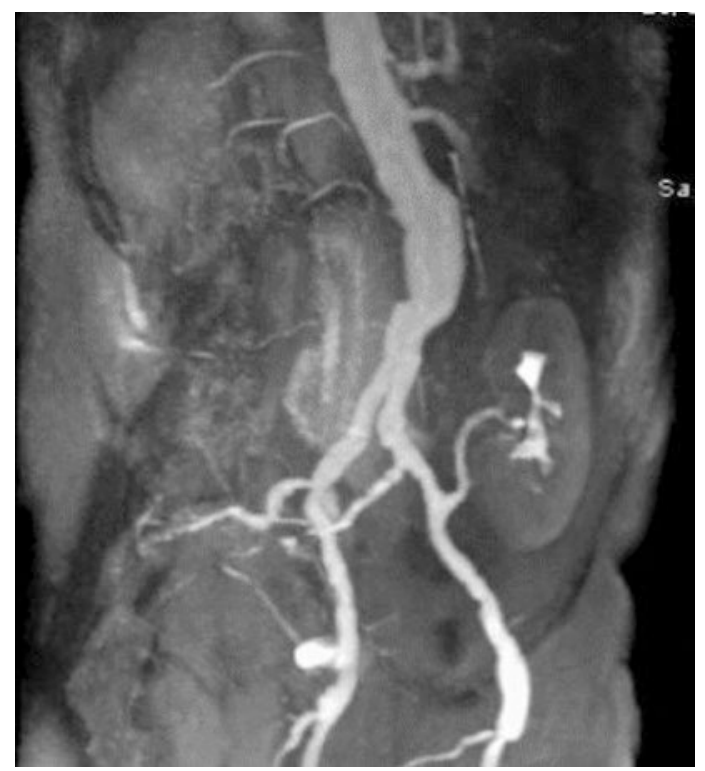

Fig. 11.3 Magnetic resonance imaging of aorto-bisiliac bypass and graft

ticular, endovascular repair before transplant does not compromise retroperitoneal tissue and vascular integrity [35]. 

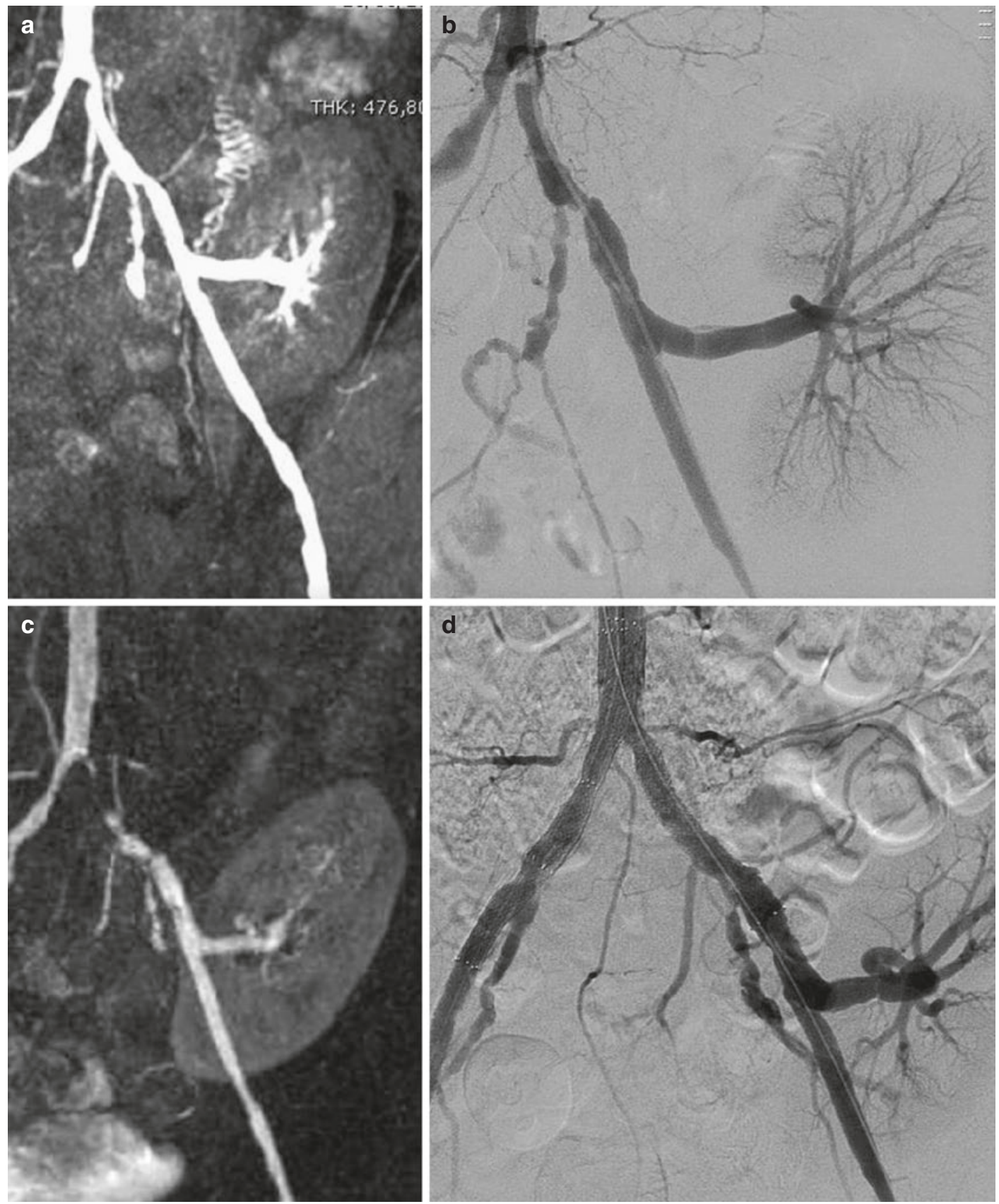

Fig. 11.4 (a) A 68-year-old female patient was referred to our center for bilateral claudication, 3 years after kidney transplant. (b) The patient was treated with bilateral angioplasty. (c) After 6 months, bilateral claudication and

moderate increase in creatinine serial levels were present. (d) The patient was treated with kissing stent. Complete resolution of claudication and restoration of kidney function. Seven years follow-up 


\section{References}

1. Lindner A, et al. Accelerated atherosclerosis in prolonged maintenance hemodialysis. N Engl J Med. 1974;290:697-701.

2. Panneton JM, et al. Aortic reconstruction in kidney transplant recipients. Ann Vasc Surg. 1996;10(2):97-108.

3 . Cron DC, et al. Aneurysms in abdominal organ transplant recipients. J Vasc Surg. 2014;59(3):594-8.

4. Galazka Z, et al. Kidney transplant in recipients with atherosclerotic iliac vessels. Ann Transplant. 1999;4:43.

5. Droupy $S$, Eschwège $P$, Hammoudi $Y$, Durrbach A, Charpentier B, Benoit G. Consequences of iliac arterial atheroma on renal transplantation. J Urol. 2006;175(3 Pt 1):1036-9.

6. Sehti GK, et al. Renovascular hypertension and acute aortic dissection in a patient with renal transplant. Am Surg. 1976;132:97-9.

7. Shons AR, et al. Renal transplantation with blood supply by axillofemoral bypass graft. Am J Surg. 1976;132:97-9.

8. Sterioff $\mathrm{S}$, et al. Temporary vascular bypass for perfusion of a renal transplant during abdominal aneurysmectomy. Surgery. 1977;82:558-60.

9. Campbell DA Jr, et al. Renal transplant protection during abdominal aortic aneurysmectomy with a pump-oxygenator. Surgery. 1981;90:559-62.

10. Lacombe M. Surgical treatment of aortoiliac aneurysms in renal transplant patients. J Vasc Surg. 2008;48(2):291-5.

11. Morris GC Jr, et al. The protective effect of subfiltration arterial pressure on the kidney. Surg Forum. 1956;6:623-4.

12. Lacombe M. Non traumatic acute obstructions of the renal artery. J Cardiovasc Surg. 1992;33:163-8.

13. Nghiem DD, et al. In situ hypothermic preservation of a renal allograft during resection of abdominal aortic aneurysm. Am Surg. 1982;48:237-8.

14. Tshomba Y, et al. Comparison of renal perfusion solution during thoracoabdominal aortic repair. J Vasc Surg. 2014;59(3):623-33.

15. Gibbons GW, et al. Aortoiliac reconstruction following renal transplantation. Surgery. 1982;91:435-7.

16. Roach DM, et al. Aortic aneurysm repair with a functioning renal transplant: therapeutic options. ANZ J Surg. 2004;74:65-7.

17. Hughes JD, et al. Renal transplant perfusion during aortoiliac aneurysmectomy. J Vasc Surg. 1985;2:600-2.

18. O'Mara CS, et al. Use of a temporary shunt for renal transplant protection during aortic aneurysm repair. Surgery. 1983;94:512-5.

19. Kashyap VS, et al. Abdominal aortic aneurysm repair in patients with renal allograft. Ann Vasc Surg. 1999;13:199-203.

20. Sawhney R, Chuter TA, Wall SD, Reilly LM, Kerlan RK, Canto CJ, Jean-Claude J, Faruqi RM. Aortic stent-grafts in patients with renal transplants. J Endovasc Ther. 2000;7(4):286-91.

21. Forbes TL, DeRose G, Kribs S, Abraham CZ, Harris KA. Endovascular repair of abdominal aortic aneu- rysm with coexisting renal allograft: case report and literature review. Ann Vasc Surg. 2001;15(5):586-90.

22. Silverberg D, Yalon T, Halak M. Endovascular repair of abdominal aortic aneurysms in the presence of a transplanted kidney. Cardiovasc Intervent Radiol. 2015;38(4):833-9.

23. Bostock IC, Zarkowsky DS, Hicks CW, Stone DH, Eslami MH, Malas MB, Goodney PP. Outcomes of endovascular aortic aneurysm repair in kidney transplant recipients: results from a National Quality Initiative. Am J Transplant. 2016;16(8):2395-400.

24. Aspelin P, Aubry P, Fransson SG, Strasser R, Willenbrock R. Berg KJ; nephrotoxicity in high-risk patients study of iso-osmolar and low-osmolar nonionic contrast media study investigators. nephrotoxic effects in high-risk patients undergoing angiography. N Engl J Med. 2003;348(6):491-9.

25. Vigneau C, Fulgencio JP, Godier A, Chalem Y, El Metaoua S, Rondeau E, Tuppin P, Bonnet F. The use of contrast media in deceased kidney donors does not affect initial graft function or graft survival. Kidney Int. 2006;70(6):1149-54.

26. Ruiz Fuentes MC, Moreno Ayuso JM, Ruiz Fuentes N, Vargas Palomares JF, Asensio Peinado C, Osuna Ortega A. Treatment with $\mathrm{N}$-acetylcysteine in stable renal transplantation. Transplant Proc. 2008;40(9):2897-9.

27. MacNeill BD, Harding SA, Bazari H, Patton KK, Colon-Hernadez P, DeJoseph D, Jang IK. Prophylaxis of contrast-induced nephropathy in patients undergoing coronary angiography. Catheter Cardiovasc Interv. 2003;60(4):458-61.

28. Maurel B, Hertault A, Sobocinski J, Le Roux M, Gonzalez TM, Azzaoui R, Saeed Kilani M, Midulla $\mathrm{M}$, Haulon S. Techniques to reduce radiation and contrast volume during EVAR. J Cardiovasc Surg. 2014;55(2 Suppl 1):123-31.

29. Carrafiello G, Ierardi AM, Radaelli A, De Marchi G, Floridi C, Piffaretti G, Fontana F. Unenhanced cone beam computed tomography and fusion imaging in direct percutaneous sac injection for treatment of type II endoleak: technical note. Cardiovasc Intervent Radiol. 2016;39(2):323.

30. Tsivian M, et al. Aortoiliac surgery concomitant with kidney transplant: a single centre experience. Clin Transpl. 2009;23:164.

31. Piquet $\mathrm{P}$, et al. Aortoiliac reconstruction and renal transplantation: staged or simultaneous. Ann Vasc Surg. 1989;3:251.

32. Gouny P, et al. Aortoiliac surgery and kidney transplantation. Ann Vasc Surg. 1991;5:26.

33. Coosemans W, et al. Renal transplantation in patients with a vascular aortoiliac prosthesis. Transplant Proc. 1999;31:1925.

34. Gill R, et al. Management of peripheral vascular disease compromising renal allograft placement and function: review of the literature with an illustrative case. Clin Tranplant. 2011;25:337-44.

35. Tozzi M, Franchin M, Soldini G, Ietto G, Chiappa C, Molteni B, Amico F, Carcano G, Dionigi R. Treatment of aortoiliac occlusive or dilatative disease concomitant with kidney transplantation: how and when? Int J Surg. 2013;11(Suppl 1):S115-9. 